\title{
Über ein Verfahren zur maschinellen, konsekutiven Eisen/Kupfer-Bestimmung .
}

\author{
Von A. Bernegger, H. Keller und R. Wenger \\ Klinisch-Chemisches Zentrallabor des Kantons St. Gallen
}

(Eingegangen am 20. Mai 1972)

Es wird ein Verfahren zur Bestimmung von Eisen und Kupfer im Serum mit Hilfe einer Autoanalyzer-Konfiguration beschrieben. Dabei wird zuerst der Eisen-Gehalt und die Eisen-Bindungskapazität, unmittelbar anschließend die Kupfer-Konzentration bestimmt. Die Umstellung erfolgt durch Wechsel der Reagenzien und des Filters im Kolorimeter, der Schlauchsatz bleibt für beide Verfahren gleich. Die analytische Präzision des Verfahrens ist befriedigend, der Probenverbrauch niedriger als bei vergleichbaren Verfahren, die Arbeitsgeschwindigkeit für den Routine-Betrieb ausreichend. Der besondere Vorteil des Verfahrens liegt in dem relativ kleinen instrumentellen Aufwand und den geringen Arbeitsanforderungen an das Bedienungspersonal.

\section{A method for the automatic, consecutive determination of iron and copper}

A method is described for the determination of iron and copper in serum with the aid of the autoanalyzer. The iron content and the iron binding capacity are determined, followed directly by the determination of the copper concentration. The changeover from iron to copper determination is achieved by changing the reagents used and the filter in the colorimeter, but the delivery tubing remains the same. The analytical precision of the method is satisfactory, a smaller sample is required than in comparable methods and the rate of throughput of samples is sufficient for routine purposes. Particular advantages of the method are the relatively limited instrumentation and its simplicity of operation.

Zur Bestimmung des Eisen-Gehaltes von Plasma oder Serum mittels eines Continuous-Flow-Verfahrens im Autoanalyzer liegen mehrere Literaturangaben vor (1-8). Ob man sich dieser Verfahren bedient, hängt von der praktisch erreichbaren Präzision und Richtigkeit, von der möglichen Verarbeitungsgeschwindigkeit, vom erforderlichen Probenvolumen, wesentlich aber auch von der Zahl der täglichen Untersuchungsaufträge $a b$, da der Einsatz kostspieliger Analysenmaschinen in einem vertretbaren Verhältnis zum erzielbaren Rationalisierungserfolg stehen muß.

Auch für die maschinelle Bestimmung des Kupfers im Serum sind Verfahren beschrieben $(9,10)$, für deren Einsatz die gleichen Kriterien gelten.

Offenbar wird die Untersuchung der Serum-EisenKonżentration nicht selten mit der Bestimmung des Kupfergehaltes gemeinsam angefordert. Deshalb wurde von KaTtERMANN und KöHrING $(11,12)$ unlängst die simultane Eisen/Kupfer-Bestimmung aus einer SerumProbe mit Hilfe einer Autoanalyzer-Konfiguration realisiert.

Die simultane Bestimmung von Eisen und Kupfer aus einer Probe hat jedoch verschiedene Nachteile:

1. Das Auftragsmuster zeigt bei vielen Laboratorien eine eindeutige Bevorzugung des Eisens vor dem Kupfer.

2. Eine häufig angeforderte Untersuchungskombination ist Eisen und Eisen-Bindungskapazität (1). Bei den für die Bestimmung der Eisen-Bindungskapazität vorbereiteten Proben ist die simultane Kupfer-Bestimmung sinnlos.

3. Auch die Zahl der Aufträge für eine isolierte KupferBestimmung ist häufig nicht so gering, als daß sie bei
Betriebs- und Arbeits-ökonomischen Überlegungen unberücksichtigt bleiben könnte.

4. Der an sich schon relativ große Materialverbrauch wird durch die simultane Eisen- und Kupfer-Bestimmung noch erheblich größer, wodurch ebenfalls Schwierigkeiten entstehen können.

5. Die apparativen Aufwendungen wachsen durch die simultane Eisen/Kupfer-Bestimmung beträchtlich.

Eine alternative Lösung, die diese Gesichtspunkte berücksichtigt, könnte darin bestehen, die Autoanalyzer-Konfiguration zur Eisen-Bestimmung auch für die nachfolgende Kupfer-Bestimmung zu benutzen, d. h. eine konsekutive Eisen/Kupfer-Bestimmung durchzuführen. Kennzeichnend für ein solches Verfahren wäre, daß die „Umrüstung" der Anlage nur einen minimalen Arbeitsaufwand erfordert, insbesondere, $\mathrm{da} B$ kein Wechsel des Pumpenschlauchsatzes notwendig ist.

Um dieses Ziel zu erreichen, wurde zur Eisen-Bestimmung ein neuer Chelat-Bildner, das 7-Brom-1,3dihydro-1-(3-dimethylaminopropyl)-5-(2-pyridyl)-2H1,4-benzodiazepin-2-on-dihydrochlorid (Hoffmann-La

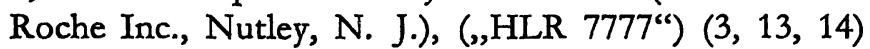
und als sehr empfindliches Reagenz für die KupferBestimmung Oxalyl-dihydrazid/Acetaldehyd ausgewählt $(9,11,12,15)$. Da beide Reagenzien bereits erfolgreich in Continuous-flow-Verfahren eingesetzt wurden, bestand die Aufgabe lediglich darin, ein geeignetes Flußdiagramm zu entwickeln.

\section{Material und Methoden}

Reagenzien für die Eisen-Bestimmung

1. $40 \mathrm{mg}$ Ascorbinsäure (Merck Art. Nr. 127) werden in $100 \mathrm{ml}$ $1 \mathrm{~mol} / 1$ Salzsäure (Mcrck Titrisol Art. Nr. 9970) gelöst und 
0,5 ml Brij-35 (Tcchnicon T 21-0110-17) 30\% zugesctzt, die Lösung wird täglich frisch zubereitet.

2. Farbrcagenz: 50,0 g Ammoniumacetat (Merck Art. Nr. 1116) werden in $500 \mathrm{ml}$ dest. Wasser gelöst, mit Eisessig (Merck Art. Nr. 90063) auf $\mathrm{pH} 5,0$ eingestellt und $1 \mathrm{~g}$ HLR 7777. zugefügt. Bis zur vollständigen Lösung wird bei Raumtemperatur gerührt, die klare Lösung erhält einen Zusatz von $0,5 \mathrm{ml}$ Brij-35 (Technicon) $30 \%$ und wird dann auf 11 mit dest. Wasser aufgefüllt.

Reagenzien für die Bestimmung der Eisen-Bindungskapazität

1. $24,2 \mathrm{mg}$ Eisen-[III]-chlorid $\cdot 6 \mathrm{H}_{2} \mathrm{O}$ (Merck Art. Nr. 3943) werden in einigen $\mathrm{ml} \mathrm{konz.} \mathrm{HCl}$ (Merck Art. Nr. 317) gelöst und mit dest. Wasser auf 11 aufgefüllt.

2. Magnesiumcarbonat zur Analyse (Merck Art. Nr. 5827).

Reagenzien für die Kupfer-Bestimmung

1. Es wird $1 \mathrm{~mol} / 1$ Salzsäure (Merck Titrisol Art. Nr. 9970) verwendet.

2. Farbreagenz: $1 \mathrm{~g}$ Oxalyldihydrazid (British Drug Houses Nr. 13094) werden in 11 dest. Wasser gelöst. Von dieser Stammlösung werden $400 \mathrm{ml}$ mit $50 \mathrm{ml}$ Ammoniak-Lösung (25\%, Merck Art. Nr: 5432) versetzt und anschließend $15 \mathrm{ml}$ Acetaldehyd (Merck Art. Nr. 4) zugesetzt.

3. $9 \mathrm{~g}$ Natriumchlorid (Fluka Nr. 71380) werden ad 11 in destilliertem Wasser gelöst und $0,5 \mathrm{ml} \mathrm{Brij-35}$ (Technicon) $30 \%$ Lösung zugegeben.

\section{Instrumentation}

Alle eingesetzten Autoanalyzer-Bauteile entstammen der „I. Generation", lediglich der Probennehmer Nr. II ist ein neueres Modell. Die Abbildung 1 zeigt das Fließ-Schema.

Der Pumpenschlauch, der das Kupfer-Reagenz dem System zuführt, bleibt während der Eisen-Bestimmung leer, d. h. er pumpt nur Luft zu einem offenen Hahn. Bei der Kupfer-Bestimmung wird durch Umlegen des 3-Wege-Hahnes das Reagenz unter Umgehung des Dialysators unimittelbar vor dem ersten Wasserbad dem Dialysat zugegeben. Das Wasserbad selbst ist kein TechniconBauteil, sondern wurde aus üblichen Labor-Thermostaten zusammengestellt.

Als Besonderheit muß noch erwähnt werden, daß die Küvette des Photometers einen Lichtweg von $50 \mathrm{~mm}$ Länge hat.

\section{Arbeitsweise}

Bevor die Untersuchungsserie beginnt, wird die Gesamtanlage $10 \mathrm{Min}$. mit Brij-Lösung $(150 \mathrm{ml} / \mathrm{l})$ gespült, und zwar sowohl die Reagenzien-Schläuche, wie der Probenschlauch. Danach werden die Reagenzien-Schläuche in die Reagenz-Flaschen, der Probenschlauch in eine Flasche mit dest. Wasser gebracht und die Anlage 20 Min. mit Reagenz und Wasser durchspült. Nach etwa 10 Min. ist die Grundlinie auf dem Schreiber stabil.

Man beginnt die Untersuchungsserie mit primären Standards $(0,5 ; 1,0 ; 2,0 ; 3,0 \mathrm{mg} / \mathrm{l})$, darauf folgen 2 Gefäße mit Wasser und anschließend 2 Gefäße mit Sekundär-Standards ( $z$. B. Hyland oder Seronorm). Dahinter befinden sich die ersten 6 Proben, in

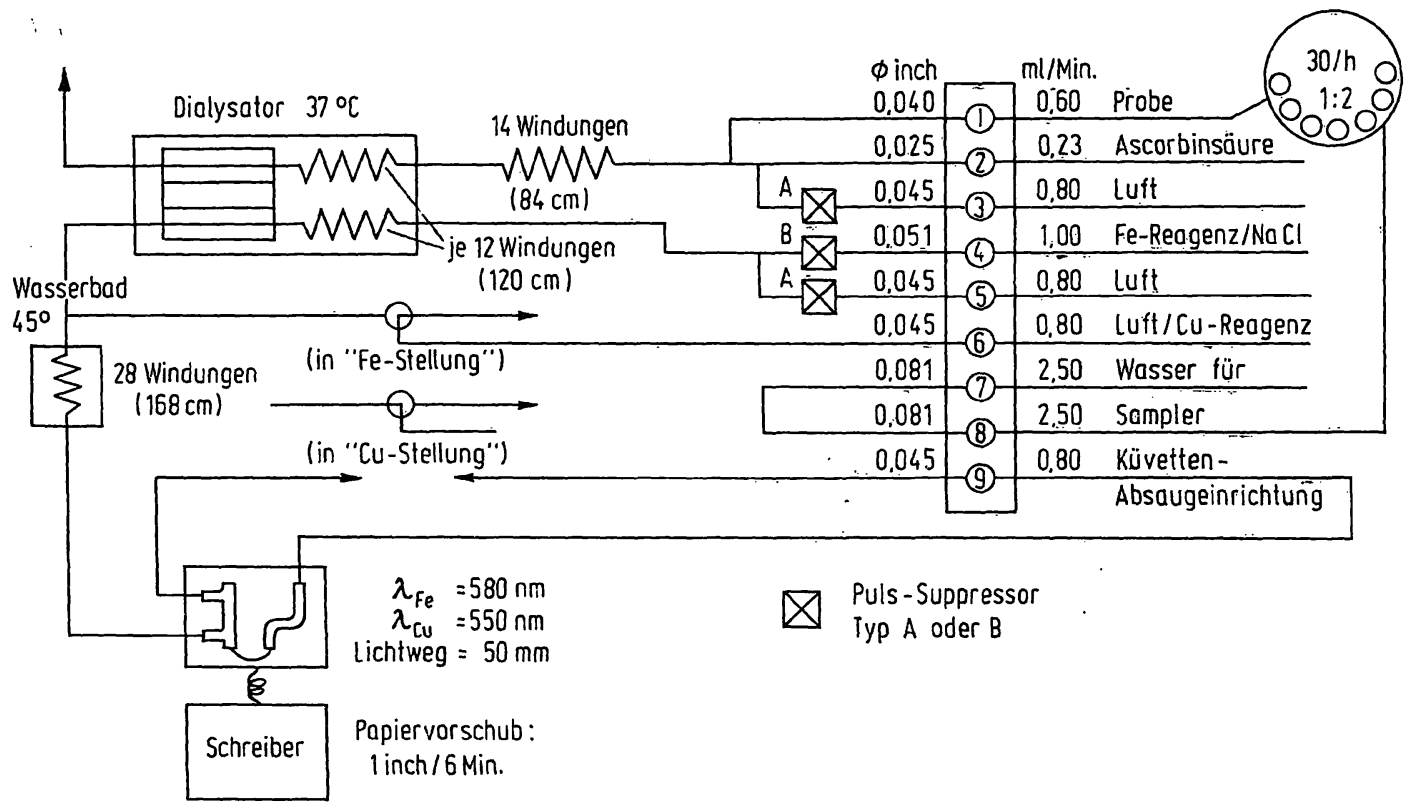

Abb. 1

Fließ-Schema des Technicon-Autoanalyzers, Einzelheiten siehe Text

Tabelle 1

Obersicht der maschinellen Eisen-Bestimmungsverfahren im Vergleich zum Ringversuch der Deutschen Gesellschaft für Klinische Chemie. 1968

\begin{tabular}{|c|c|c|c|c|c|}
\hline Jahr & Autor & Fe-Ligand & $\begin{array}{c}\text { Proben-Schlauch } \\
\text { Förder-Leistung } \\
\text { [ml/min] }\end{array}$ & $\begin{array}{l}\text { Sampler } \\
\text { Takt/h }\end{array}$ & $\begin{array}{l}\text { Proben- } \\
\text { Verbrauch } \\
\text { etwa ml }\end{array}$ \\
\hline 1963 & LEPPLA (4) & 1.10-Phenanthrolinsulfonat & 1,2 & $20-40$ & $3,6-2,4$ \\
\hline 1965 & ZAK (8) & Bathophenanthrolinsulfonat ' & 2,0 & 40 & 3,0 \\
\hline 1965 & YOUNG (6) & Tripyridyltriazin & 1,6 & 60 & 1.6 \\
\hline 1967 & SCHMIDT (5) & Dipyridyl-p-methoxyphenyl-pyridin & 1,2 & 60 & 1,2 \\
\hline 1970 & KLEIN (13) & Benzodiazepinon & 1,0 & $.20(2: 1)$ & 2,0 \\
\hline 1970 & FÜHR (2) & Bathophenanthrolinsulfonat & 0,6 & 60 & 0,6 \\
\hline 1971 & KATTERMANN (12) & Bathophenanthrolinsulfonat & 1,2 & 30 & 1,4 \\
\hline 1972 & SG, diese Arbeit & Benzodiazepinon & $(+1,2$ f. Cu $)$ & $(70 / 50)$ & $(+1,4=2,8)$ \\
\hline 1968 & STAMM (16) Ringvers. 1968 & $\begin{array}{l}\text { Phenanthrolin, Bathophenanthrolin, } \\
\text { DMPP }\end{array}$ & $\begin{array}{l}0,6 \\
-\end{array}$ & $\begin{array}{c}30(1: 2) \\
-\end{array}$ & $\underline{0,4}$ \\
\hline
\end{tabular}




\title{
Moderne digitale Meßtechning rationalisiert
}

\author{
photometrische Analingean
}

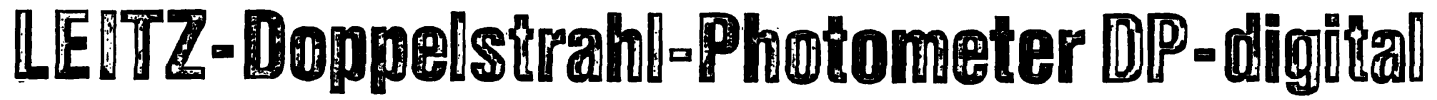

Mit dem neuen LEITZ-Doppelstrahl-Photometer DP-digital werden Ihre MTA oder Ihr Laborant die Routinearbeit problemloser bewältigen. Sie können sich ganz auf die Vorbereitung der Analysen konzentrieren-die Durchführung der Analyse übernimmt das Photomẹter. Und das Ergebnis erscheint in Bruchteilen von Sekunden in großen Leuchtzahlen: 4-ziffrig, exakt, dezimalstellenrichtig und ablesefehlerfrei.
Einige weitere technische Hinweise:

Automatischer Abgleich

Automatische Meßwertbildung

Automatische Umschaltung der Lichtquellen

Automatische Verstärkungsregelung

Digitale Anzeige von Transmission,

Extinktion oder Konzentration

Küvettenautomatik für Enzymkinetiken

Anschlußmöglichkeiten für Drucker, Schreiber,

Tischrechner

Proben- und Analysenautomatik usw.

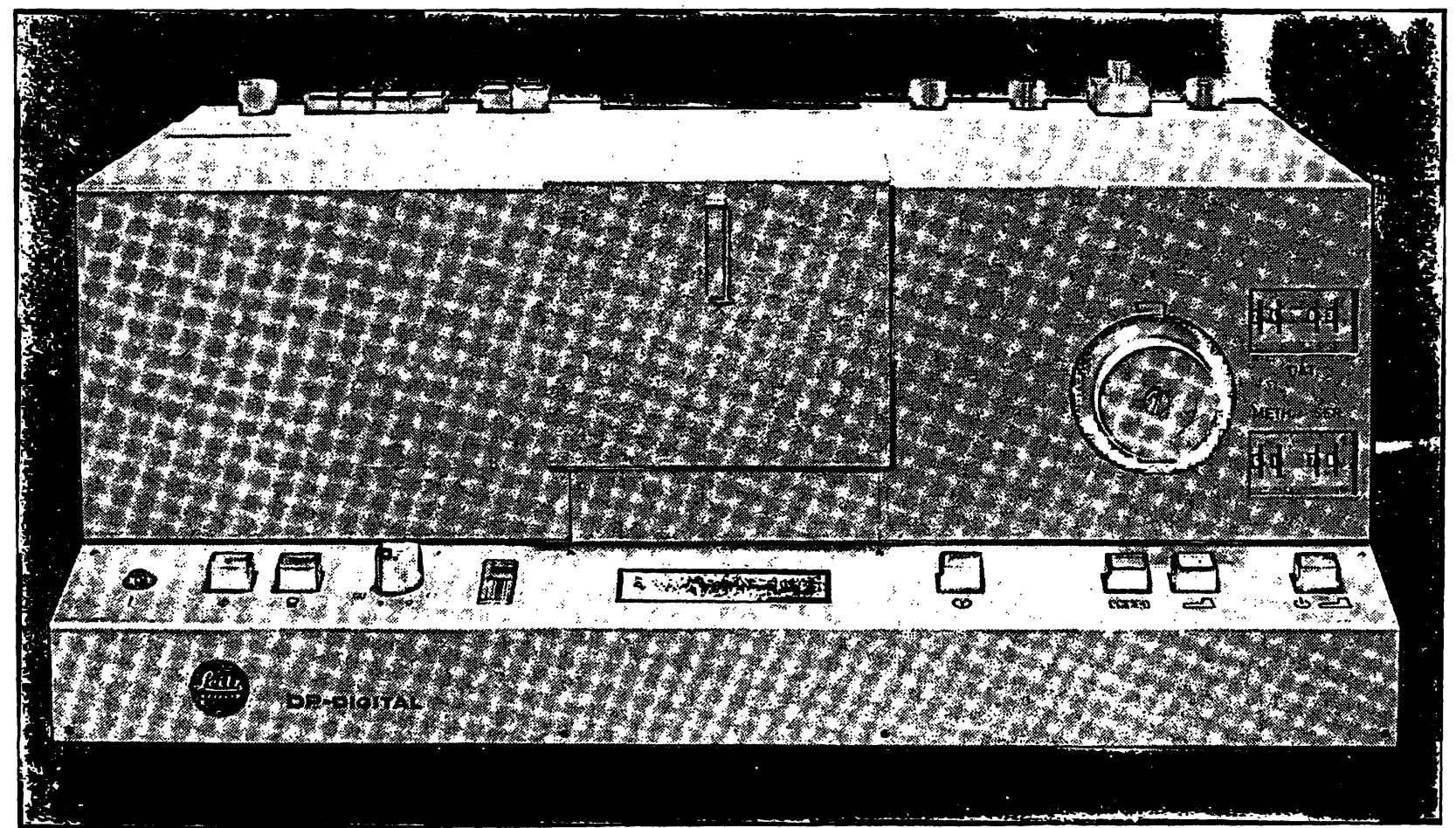

ERNST LEITZ GmbH 6330 Wetzlar

Informationsdienst DP 45
Ich bin an Ihrem neuen Doppelstrahl-Photometer DP-digital interessiert und bitte um:

den ausführlichen Prospekt [I] Name:

einen Kostenvoranschlag 1] Ort: ( )

Demonstration $\square$ Straße: 
Sigma freut sich anzubieten

Creatin Phosphokinase

Sorgfältig standardisierte Ampullen

fïr. Einzel- oder Serienbestimmungen

im Serum oder anderen Flüssigkeiten bei $340 \mathrm{~nm}$ nach dem Sigma Technical Bulletin No. 45-UV

Ungewöhnlich einfach, bequem und schnell! 1 . Wasser und $0,1 \mathrm{ml}$ Serum zugeben, „äquilibrieren“

2. Anfangsextinktion ablesen

3. Nach 5 Minuten 2. Extinktion ablesen.

Das ist alles!

Fordern Sie Sigma Technical Bulletin No. 45-UV an oder bestellen Sie:

Besfell-Nr. 45-1 Ampullen für Einzel-Bestimmungen, 10 Ampullen für 10 Bestimmungen $\$ 11,50$

Besfell-Nr. 45-5 Ampullen für fünf Bestimmungen, 10 Ampullen für 50 Bestimmungen $\$ 42,50$

Preise einschließlich Versand durch Luftpost an jeden Ort der Welt

\section{SIGMA CPK Verfahren}

Sigma hat wahrscheinlich mehr Entwicklungsarbeit für CPK aufgewandt als jede andere Firma. Diese Untersuchungen, verbunden mit Sigmas Spitzenposition als derWelt größter Hersteller von Forschungs-Biochemica, versetzen uns in die Lage, daß wir Ihnen für fast jedes Gerät mehrere äußerst zuverlässige Methoden anbieten können :

No. 661 - Kolorimetrisches Verfahren: Erfordert 0,6 ml Serum.

ATP + Creatin $\rightarrow$ ADP + Phosphocreatin $+P_{i}$. Gemessen wird $P_{i}$ nach Fiske und Subbarow.

Mit Fiske und Subbarow Reagenzien

Ohne Fiske und Subbarow Reagenzien

Besteck Nr. 661-PA 25 Bestimmungen $\$ 13,00$

Besteck Nr. 661-A 25 Bestimmungen $\$ 6,00$

Besteck Nr. 661-PB 100 Bestimmungen $\$ 32,25$

Besteck Nr. 661-B 100 Bestimmungen $\$ 19,00$

No. 520 - Kolorimetrisches Verfahren: Erfordert nur 0,01 $\mathrm{ml}$ Serum.

ADP + Phosphocreatin $\rightarrow$ ATP + Creatin. Gemessen wird das freigesetzte Creatin durch Reaktion mif Diacetyl und Naphthol. Sehr empfindlich.

Besteck Nr. 52025 Bestimmungen $\$ 18,75$

Besteck Nr. 520-C 100 Bestimmungen $\$ 44,75$

No. 80-F Fluorometrisches Verfahren: Erfordert nur $0,01 \mathrm{ml}$ Serum.

ADP + Phosphocreatin $\rightarrow$ ATP + Creatin. Gemessen wird die Fluoreszenz von Creatin in Gegenwart von Ninhydrin.

Besteck No. 80-F 20 Bestimmungen $\$ 17,75$

Besteck No. 80F-C 100 Bestimmungen $\$ 49,50$

No. 40-UV - Kinetisches UV.Verfahren bei $340 \mathrm{~nm}$. Erfordert 0,2 $\mathrm{ml}$ Serum.

ATP + Creatin $\rightarrow$ ADP + Phosphocreatin. Gemessen wird ADP im gekoppelten Optischen Test in Gegenwart von Pyruvatkinase, LDH und NADH auf Grund des Extinktionsabfalls bei $340 \mathrm{~nm}$ infolge Oxidation des NADH.

Besteck No. 40-A 15 Bestimmungen $\$ 25,00$

Besteck No. 40-C 90 Bestimmungen $\$ 138,50$

Besteck No. 40-B 45 Bestimmungen $\$ 70,00$

No. 45-UV - UV-Stat-Verfahren bei $340 \mathrm{~mm}$.

Alle Reagenzien sind in Ampullen für Einzel- oder Serienbestimmungen vorgegeben. Nach Zugabe von Wasser und $0,1 \mathrm{ml}$ Serum wird äquilibriert. Die Extinktion wird zu Beginn und nach 5 Minuten abgelesen. Ungewöhnlich einfach, bequem und schnell.

Bestell-No. 45-1 Ampullen für Einzel-Bestimmungen 10 Ampullen für 10 Bestimmungen $\$ 11,50$

Bestell-No. 45-5 Ampullen für fünf Bestimmungen 10 Ampullen für 50 Bestimmungen $\$ 42,50$

No. 910 - Automatisiertes kolorimetrisches Verfahren.

Eine automatisierte Version unseres Verfahrens No. 520. Obwohl dies wahrscheinlich das genaueste zur Zeit erhältliche automatisierte Verfahren ist, führen wir jetzt mit unserem verbesserten Verfahren No. 911, in welchem das $\alpha-N a p h t h o l-R e a g e n z$ durch das haltbarere Orcin ersetzt wird, eine Feldstudie durch. Die Preise sind dieselben wie für No. 910. Anfragen oder Bestellungen werden erbełen. Selbstverständlich garantieren wir für Ihre Zufriedenheit.

Besteck-No. 910-A 300 Bestimmungen $\$ 104,00$

Besteck-No. 910-B 1500 Bestimmungen $\$ 381,50$

Ferner ist erhältlich: CPK Bezugsenzym, ein sorgfältig getestetes Präparat, welches als Kontrolle oder Bezugsgröße für die verschiedenen von Sigma angebotenen CPK-Verfahren benutzt werden kann. Je nach Bedarf in zwei Größen erhältlich. Aktivität: 30-50 Sigma-Einheiten pro $\mathrm{ml}$ nach Auffüllen mit Wasser.

CPK-1 Ampullen für $1 \mathrm{ml} 10$ Ampullen $\$ 8,75$

CPK-2 Ampullen für $2 \mathrm{ml} 10$ Ampullen $\$ 12,50$

Wir übernehmen sämtliche normalen Versandkosten für diese Reagenzien - an jeden Ort der Welt.

Sigma Technical Bulletins für die oben aufgeführten Methoden sind auf Anfrage gratis erhältlich.

Es ist ein Vergnügen, Geschäfte mit Sigma zu machen

Bestellen Sie direkt - R-Gespräch von überall her in der Welt. Tagsüber von Haus zu Haus, 314-771-5750 (einschließlich samstags und sonntags bis 13 Uhr). Nachts von Person zu Person, Dan Broida, 314-993-6418 TWX (Fernschreiber) Tag und Nacht: Rückantwort 910-761-0593

Sigma-Reagenzien sind in der ganzen Welt durch den Fachhandel oder direkt aus St. Louis beziehbar. Telegramme: SIGMACHEM, St. Louis, Missouri

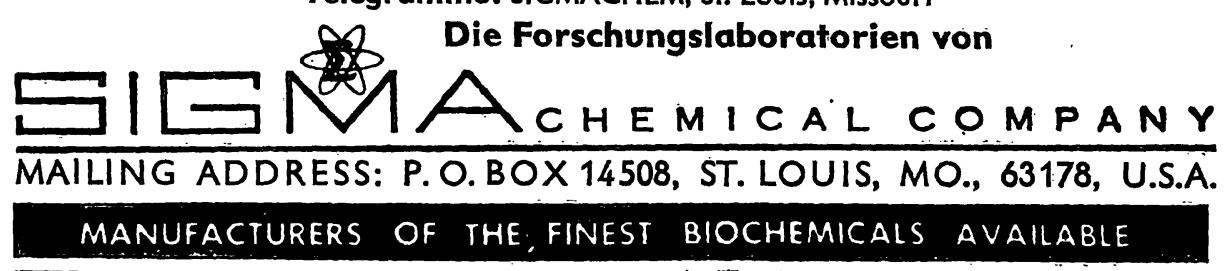

SIGMA LONDON Chem Vertreten durch

SIGMA LONDON Chem. Co., Ltd. - Norbiton Station Yard, Kingston-upon-Thames

Surrey, KT2 7BH, England. Phone 01-549-3171 (Reverse Charges)

SIGMA ISRAEL Chem. Co. Lłd. - P. O. Box 25011, Tel-Aviv 61250, Israel Phone: (03) 760654 (Reverse Charges) 
der 7. Position folgt ein Sekundär-Standard, die 8. bis 14. Position nehmen wieder Untersuchungsseren ein usf. Zwischen den Proben befindet sich an unbekannter, täglich wechselnder Stelle die Qualitätskontrolle. Wenn die Serie der Eisen-Bestimmung durchgelaufen ist, werden die in der Zwischenzeit vorbereiteten Becher der Proben für die Eisen-Bindungskapazität in den Probenteller gesetzt.

\section{Vorbereitung zur Eisen-Bindungskapazität}

$0,5 \mathrm{ml} \mathrm{Serum} \mathrm{werden} \mathrm{mit} 1 \mathrm{ml} \mathrm{FeCl}{ }_{3}$-Lösung versetzt $(=10 \mathrm{mg} / 1$ Eisen), gut durchgeschüttelt und $5 \mathrm{Min}$. bei Raumtemperatur inkubiert. Anschließend werden etwa $0,05 \mathrm{~g} \mathrm{MgCO}_{3}$ an Substanz zugegeben und die Suspension $30 \mathrm{Min}$. auf einer Schüttelmaschine geschüttelt, anschließend wird zentrifugiert (2 Min.; $10000 \mathrm{~g}$ ). Etwa $1 \mathrm{ml}$ Uberstand werden mit einer Marburg-Pipette in einen Autoanalyzer-Becher transferiert, danach erfolgt die Eisen-Bestimmung wie oben geschildert.

\section{Umstellung zur Kupfer-Bestimmung}

Nachdem alle Eisen- und Eisen-Bindungsbestimmungen durchgeführt sind, werden die Reagenz-Schläuche und der Probenschlauch 10 Min. mit Wasser durchspült. Danach kommt der EisenReagenz-Schlauch in das Vorratsgefäß für NatriumchloridLösung, die Kupfer-Reagenz-Linie wird eingeschaltet und der Kupfer-Reagenz-Schlauch kommt ins Kupfer-Reagenz. Schließlich werden die Filter des Kolorimeters gewechselt. Es werden nun $15 \mathrm{Min}$. Reagenz durch die Reagenzien-Schläuche, bzw. Wasser durch den Probenschlauch gesaugt. Anschließend beginnt die Bestimmung von primären Standards $(0,5 ; 1,0 ; 2,0$; $3,0 \mathrm{mg} / \mathrm{l})$ danach folgen 2 Becher mit Wasser, danach 2 gleiche Sekundär-Standards (Hyland oder Seronorm) und schließlich die ersten 6 Proben. Der Arbeitsrhythmus ist der gleiche wie bei der Eisen-Bestimmung.

\section{Ergebnisse und Diskussion}

Die Ergebnisse einer primären Eichreihe zeigt die Abbildung 2a für die Eisen- und $2 b$ für die KupferBestimmung. Für die Eisen-Bestimmung konnten wir im wesentlichen die Ergebnisse von KLEIN und Mitarbeiter (3) bestätigen, die einen Variationskoeffizienten von 3,5\% gefunden haben. Die Streuung, berechnet aus den Qualitätskontrollversuchen der Monate Januar, Februar und März 1972 betrug $s= \pm 40,6 \mu \mathrm{g} / \mathrm{l}$ bei einem $\bar{x}$ von $1030,8 \mu \mathrm{g} / \mathrm{i}$, was einem Variationskoeffizienten von $\mathrm{VK}=3,94 \%$ entspricht.

Bei der Kupfer-Bestimmung überprüften wir die Angaben von Summers (9), der eine Streuung zwischen 14 und $23 \mu \mathrm{g} / 1$ angibt, was im günstigsten Fall einem

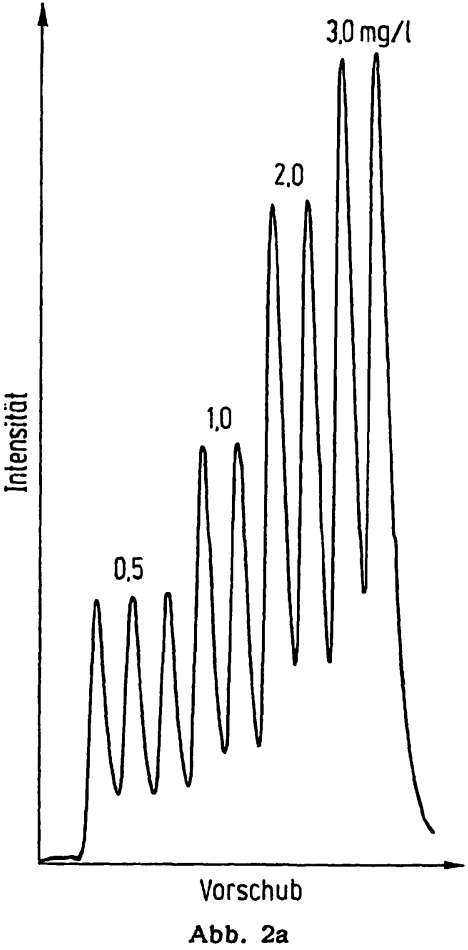

Eichreihe für die Eisen-Bestimmung, die Standard-Lösungen enthalten 0,$5 ; 1,0 ; 2,0 ; 3,0 \mathrm{mg} / 1$ Eisen

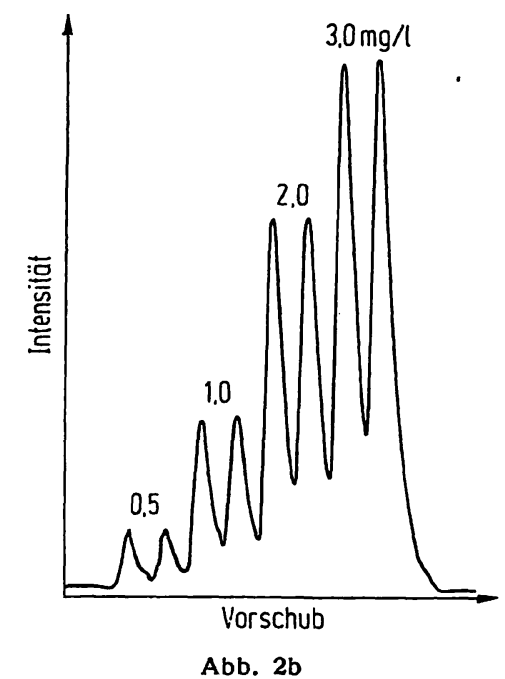

Eichreihe für die Kupfer-Bestimmung, die Standard-Lösungen enthalten 0,$5 ; 1,0 ; 2,0 ; 3,0 \mathrm{mg} / 1 \mathrm{Kupfer}$

Fortsetzung Tabelle 1

\begin{tabular}{|c|c|c|c|}
\hline$[\mu \mathrm{g} / 1]$ & $\mathbf{s}$ & VK\% & Bemerkungen \\
\hline $200-3000$ & 30 & $3,0(?)$ & Konventionelle Bauteile, Dialyse, range expander \\
\hline 1000 & 48 & 4,8 & Konventionelle Bauteile, Dialyse, range expander \\
\hline 1000 & 23 & 2,3 & Konventionelle Bauteile, Dialyse \\
\hline 1000 & 47,3 & 4,7 & Konventionelle Bauteile, Dialyse, range expander \\
\hline 830 & 29 & 3,5 & Konventionelle Bauteile, Dialyse \\
\hline 820 & 30,5 & 3,7 & $\begin{array}{l}2 \text { hintereinander geschaltete Fotometer u. } 2 \text { Schreiber (Hg-Lampe u. Linearisierungl) keine Dialyse } \\
\text { (keine Enteiweißung) }\end{array}$ \\
\hline 910 & 89 & 9,7 & Simultane $\mathrm{Fe} / \mathrm{Cu}$-Bestimmung, 2 Kolorimeter, 2 Dialysatoren, Doppelschreiber \\
\hline $\begin{array}{l}1030,8 \\
873\end{array}$ & $\begin{array}{l}40,6 \\
69\end{array}$ & $\begin{array}{l}3,9 \\
13 / 14\end{array}$ & $\begin{array}{l}\text { Konsekutive } \mathrm{Fe} / \mathrm{Cu}-\mathrm{Bestimmung} \text {, konventionelle Bauteile } \\
\text { unterschledliche, auch manuelle Verfahren }\end{array}$ \\
\hline 783 & 72 & & \\
\hline
\end{tabular}


Variationskoeffizienten von $\mathrm{VK}=1,15 \%$ und im ungünstigsten Fall VK $=1,87 \%$ entspricht. Die von uns festgestellte Streuung über eine Periode von 3 Monaten betrug $\mathrm{s}= \pm 18,8 \mu \mathrm{g} / \mathrm{l}$ bei einem $\overline{\mathrm{x}}$ von $1137,7 \mu \mathrm{g} / \mathrm{l}$, was einem Variationskoeffizienten von $\mathrm{VK}=1,66 \%$ entspricht.

Bei beiden Verfahren ist die Wiederfindung in Übereinstimmung zu den Literaturangaben voll befriedigend, ihre Abweichungen vom Sollwert entsprechen der Streuung.

Während die Verfahren zur Kupfer-Bestimmung relativ unkritisch sind und durch die Bestimmung des Caeruloplasmin ein zweiter diagnostisch gleichwertiger Parameter zur Verfügung steht, bereiten Eisen-Bestimmungen häufig Schwierigkeiten. Sowohl beim manuellen wie maschinellen Verfahren muß häufig eine unbefriedigende Präzision von Tag zu Tag festgestellt werden. Besonders aufschlußreich war in dieser Hinsicht die Feststellung von STAMM und BürTNER (16), wonach beim Ringversuch der Deutschen Gesellschaft für Klinische Chemie 1968 bei der Eisen-Bestimmung Variationskoeffizienten von 13 und $14 \%$ gefunden wurden, unabhängig, welche Verfahren angewandt wurden. Die in der Tabelle 1 wiedergegebenen Streuungen, resp. Variationskoeffizienten, sind in der Regel wesentlich niedriger, was in einem offensichtlichen, aber durchschaubaren Widerspruch zu den Resultaten des Ringversuches steht.

Die von uns mitgeteilten Werte für die Kupfer- wie auch für die Eisen-Bestimmung wurden deshalb einer längeren Beobachtungsperiode aus den Protokollen der täglichen Routine entnommen, die von wechselndem Personal ohne Kenntnis der Untersuchungsabsichten und des sogenannten „wahren Wertes" registriert worden war. Diese Werte wurden ohne Selektion mit Hilfe eines programmierbaren Tischrechners verarbeitet. Sie dürften daher auch von anderen Laboratorien und auch unter den Bedingungen eines Ringversuches reproduzierbar sein.

Unabhängig von den subjektiven Faktoren, die bei einzelnen Autoren zur Angabe besonders hoher Präzision einzelner Verfahren führen, zeigt die Tabelle 1 , $\mathrm{da} \beta$ in den etwa 10 Jahren, seit dem die maschinelle
Eisen-Bestimmung zur Verfügung steht, offenbar keine nennenswerten Verbesserungen erzielt wurden: trotz Einsatz verschiedener Eisen-Liganden ließen sich Streuungs- resp. Variationskoeffizienten nicht eindeutig verkleinern. Da immer wieder neue Anordnungen zur maschinellen Eisen-Bestimmung beschrieben wurden, scheinen die vorangegangenen den klinischen Bedürfnissen nicht entsprochen zu haben (16). Die Einführung des Benzo-diazepinons, dessen molarer Extinktionskoeffizient $\left(\varepsilon_{580}=17400 \mathrm{~cm}^{2} / \mathrm{mol}\right)$ deutlich niedriger ist als der anderer Komplexbildner $(5,14)$, hat den Vorteil, daß unmittelbar in das Chelat-bildende Reagenz dialysiert werden kann und keine Hydrochinonlösung mit beschränkter Haltbarkeit erforderlich ist. Dadurch ist es möglich, ohne Verstärker („tange expander") die Signale des Photometers aufzuzeichnen. Die Erwärmung des Dialysats auf $45^{\circ} \mathrm{C}$ ist für die Kupfer-Bestimmung zweckmäßig, für die Eisen-Bestimmung nicht erforderlich, jedoch unter den angegebenen Umständen auch nicht störend. Ein Umstand, der wichtig ist, um die Konfiguration der Analysenmaschine für beide Bestimmungen unverändert halten zu können. Der Einsatz des.. Dialysators ist bei der Mehrzahl der maschinellen Konfigurationen üblich. Die Vorteile, ohne Dialysator zu arbeiten (2), werden mit einem beträchtlichen instrumentellen Mehraufwand erkauft. Da nach den hier vorliegenden Erfahrungen die Membranen relativ lang funktionsfähig sind, bestand kein Grund, auf die Dialyse zu verzichten.

Ein wesentlicher Gesichtspunkt bei der Konzeption der hier beschriebenen Anlage war der Wunsch, mit einem Minimum an Untersuchungsmaterial auszukommen. Wie aus der Tabelle hervorgeht, ist dies auch gelungen. Für Eisen- und Kupfer-Bestimmungen werden nur je $0,4 \mathrm{ml}$ Serum benötigt.

$\mathrm{Da}$ die hier beschriebene Anlage aus Einzelteilen zusammengesetzt ist, die zum Teil über 10 Jahre im Gebrauch waten und die nicht mehr der modernen Technologie entsprechen, kann man erwarten, daß mit Bauteilen des Autoanalyzers der II. Generation noch weitere Verbesserungen hinsichtlich Durchsàtz-Geschwindigkeit, Reagenz- und Materialverbrauch sowie analytischer Präzision zu erzielen wären.

\section{Literatur}

1. Friedman, H. S. und C. S. Cheek, Clin. Chim. Acta, Amsterdam 31, 315 (1971). - 2. Fürr, J. und E. Stary, Ärztl. Lab. 16, 244 (1970). - 3. KleiN, B., N. Kleinman und R. L. SEARCY, Clin. Chem., New York 16, 495 (1970). - 4. Leppla, W., W. Brokate und H. E. Keller, Internat. Techn. Sympos. Automat. Analyt. Chemie, 225 (1963). - 5. Schmidt, R., W. Weis, V. Klingmüller und H. J. Staudinger, diese Z. 5, 304 (1967). 6. Young, D. S. und J. M. Hrcks, J. Clin. Pathol. (London) 18, 98 (1965). - 7. ZAK, B. und E. Epstern, Internat. Techn. Sympos. Automat. Analyt. Chemie, 485 (1964). - 8. ZAK, B. und E. Epstern, Clin. Chem., New York 11, 641 (1965). - 9. Sumamers, R. M., Anal. Chem. 32, 1903 (1960). - 10. Summers, R. M.,
R. B. Mefrert und H. R. Lirtueton, Internat. Techn. Sympos. Automat. Analyt. Chemie, (1964). - 11. KattermanN, R. und B. Köhring, Internat. Techn. Sympos. Automat. Analyt. Chemie, (1971). - 12. KattermanN, R. und B. Köhring, diese Z. 9, 391 (1971). - 13. Klein, B., L. B. Lucas und R. L. Searcy, Clin. Chim. Acta, Amsterdam 26, 517 (1969). - 14. KleIN, B., B. K Weber, L. Lucas, J. A. Foreman und R. L. Searcy, Clin. Chim. Acta, Amsterdam 26, 77 (1969). - 15. Beale, R. N. und D. CROFT, J. Clin. Pathol., London 17, 260 (1964). - 16. StamM, D. und H. BütTNER, diese Z. 7, 393 (1969). - 17. DeGGAU, E., F. KRöHNKE, K. E. Schnalke, H. J. Staudinger und W. Weis, diese $Z$. 3, 102 (1965).
Prof. Dr. H. Keller Kantonspital $\mathrm{CH}=9000$ St. Gallen (SG) 